\title{
New Constructions of Nullnorms on Bounded Lattices
}

\author{
Jiajia Xie, Wei Ji* \\ College of Science, Guilin University of Technology, Guilin, China \\ Email:949158765@qq.com, *javeey@163.com
}

How to cite this paper: Xie, J.J. and Ji, W. (2021) New Constructions of Nullnorms on Bounded Lattices. Journal of Applied Mathematics and Physics, 9, 1-10. https://doi.org/10.4236/jamp.2021.91001

Received: December 8, 2020

Accepted: December 27, 2020

Published: December 30, 2020

Copyright $\odot 2021$ by author(s) and Scientific Research Publishing Inc. This work is licensed under the Creative Commons Attribution International License (CC BY 4.0).

http://creativecommons.org/licenses/by/4.0/

\begin{abstract}
We propose two more general methods to construct nullnorms on bounded lattices. By some illustrative examples, we demonstrate that the new method differ from the existing approaches.
\end{abstract}

\section{Keywords}

Nullnorm, Triangular Norm, Triangular Subnorm, Bounded Lattice

\section{Introduction}

The notions of triangular norms ( $\mathrm{t}$-norms for short) and triangular conorms ( $\mathrm{t}$-conorms for short) were introduced by Schweizer and Sklar [1]. Nullnorms are generalizations of triangular norms and triangular conorms with a zero element in the interior of the unit interval, and have to satisfy some additional constraints. Nullnorms are important from a theoretical viewpoint but also because of their numerous potential applications, such as expert systems, fuzzy quantifiers, neural networks, fuzzy logic [2]. The constructions of nullnorms were first studied on the unit interval [2]-[9]. In the subsequent studies, the interval has extended to bounded lattices [10] [11] [12].

Some constructions of nullnorms on bounded lattices were demonstrated in previously papers. Based on the existence of t-norms and t-conorms on an arbitrary bounded lattice, Karaçal et al. [10] proposed three construction methods of nullnorms on bounded lattices with an arbitrary zero element $a \in L \backslash\{0,1\}$. Subsequently, Ümit Ertuğrul [11] proposed two construction methods of nullnorms on bounded lattices, which can be recognized as generalizations of two construction methods proposed in [10].

In this paper, we propose two more general construction methods of nullnorms on an arbitrary bounded lattice. The present study is organized as follows: 
In Section 2, we recall some basic concepts and show some existing constructions of nullnorms on an arbitrary bounded lattice. In Section 3, we introduce the notions of t-subnorm and t-subcnonorm. By using these operations, we propose new methods to obtain nullnorms on $L$ under some additional constraints and their characteristics are examined. Finally, this summarization can be found in Section 4.

\section{Preliminaries}

A lattice is a partially ordered set $(L, \leq)$ in which each two-element subset $\{x, y\}$ has an infimum, denoted as $x \wedge y$, and a supremum, denoted as $x \vee y$. A bounded lattice $(L, \leq, 0,1)$ is a lattice that has the bottom and top elements written as 0 and 1 , respectively. We denote $(L, \leq, 0,1)$ simply by $L$ in this article.

Let $(L, \leq, 0,1)$ be a bounded lattice and $V_{1}, V_{2}: L^{2} \rightarrow L$ be two binary operations on $L$, we can define a partial order:

$$
V_{1} \leq V_{2} \Leftrightarrow V_{1}(x, y) \leq V_{2}(x, y) \text { for all } x, y \in L .
$$

Given a bounded lattice $(L, \leq, 0,1)$ and $a, b \in L, a \leq b$, a subset $[a, b]$ of $L$ is defined as $[a, b]=\{x \in L \mid a \leq x \leq b\}$. Similarly, denote $[a, b)=\{a \leq x<b\}$, $(a, b]=\{x \in L \mid a<x \leq b\}$ and $(a, b)=\{x \in L \mid a<x<b\}$. If $a$ and $b$ are incomparable, we use the notation $a \| b$. The set of all elements which are incomparable with $a$ are denoted by $I_{a}$.

Definition 2.1. ([13] [14]) Let $(L, \leq, 0,1)$ be a bounded lattice. An operation $T: L^{2} \rightarrow L$ is called a triangular norm (t-norm for short) if it is commutative, associative, increasing with respect to both variables and has the neutral element $1 \in L$ such that $T(1, x)=x$ for all $x \in L$.

Definition 2.2. ([13] [14]) Let $(L, \leq, 0,1)$ be a bounded lattice. An operation $S: L^{2} \rightarrow L$ is called a triangular conorm (t-conorm for short) if it is commutative, associative, increasing with respect to both variables and has the neutral element $0 \in L$ such that $S(0, x)=x$ for all $x \in L$.

Definition 2.3. ([15]) Let $(L, \leq, 0,1)$ be a bounded lattice. An operation $F: L^{2} \rightarrow L$ is called a t-subnorm on $L$ if it is commutative, associative, increasing with respect to both variables and $F(x, y) \leq x \wedge y$ for all $x, y \in L$.

Definition 2.4. ([15]) Let $(L, \leq, 0,1)$ be a bounded lattice. An operation $R: L^{2} \rightarrow L$ is called a t-subconorm on $L$ if it is commutative, associative, increasing with respect to both variables and both $R(x, y) \geq x \vee y$ for all $x, y \in L$.

Proposition 2.5. ([15]) If $F_{1}: L^{2} \rightarrow L$ is a t-subnorm on a bounded lattice $L$, then $T: L^{2} \rightarrow L$ defined by

$$
T(x, y)= \begin{cases}F_{1}(x, y), & \text { if }(x, y) \in(L \backslash\{1\})^{2} \\ x \wedge y, & \text { otherwise }\end{cases}
$$

is a t-norm on $L$.

Dually, if $R_{1}: L^{2} \rightarrow L$ is a t-subconorm on a bounded lattice $L$, then 
$S: L^{2} \rightarrow L$ defined by

$$
S(x, y)= \begin{cases}R_{1}(x, y), & \text { if }(x, y) \in(L \backslash\{0\})^{2} \\ x \vee y, & \text { otherwise }\end{cases}
$$

is a t-conorm on $L$.

Definition 2.6. ([10]) Let $(L, \leq, 0,1)$ be a bounded lattice. A commutative, associative, non-decreasing in each variable function $V: L^{2} \rightarrow L$ is called a nullnorm if an element $a \in L$ exists such that $V(x, 0)=x$ for all $x \leq a$ and $V(x, 1)=x$ for all $x \geq a$.

It is easy to see that $V(x, a)=a$ for all $x \in L$, and thus $a$ is the zero element for $V[10]$.

Proposition 2.7. ([16]) Let $(L, \leq, 0,1)$ be a bounded lattice and $V: L^{2} \rightarrow L$ be a nullnorm on $L$ with the zero element a. Then, $[(i)]$

(i) $\left.V\right|_{[0, a]^{2}}:[0, a]^{2} \rightarrow[0, a]$ is a t-conorm on $[0, a]$;

(ii) $\left.V\right|_{[a, 1]^{2}}:[a, 1]^{2} \rightarrow[a, 1]$ is a t-norm on $[a, 1]$.

Let $(L, \leq, 0,1)$ be a bounded lattice and $a \in L \backslash\{0,1\}$. Let $T:[a, 1]^{2} \rightarrow[a, 1]$ be a t-norm on $[a, 1]$ and $S:[0, a]^{2} \rightarrow[0, a]$ be a t-conorm on $[0, a]$. Based on the knowledge of the existence of t-norms and t-conorms on an arbitrary given bounded lattice, many construction methods of nullnorms were presented in previous papers. Generally speaking, these construction methods on an arbitrary bounded lattice under no additional constraints can be divided into two groups. One is $V_{a}^{(T, S)}(x, y): L^{2} \rightarrow L$ proposed by Karaçal et al. in [10], which is defined as

$$
V_{a}^{(T, S)}(x, y)= \begin{cases}S(x, y), & \text { if }(x, y) \in[0, a]^{2} \\ T(x, y), & \text { if }(x, y) \in[a, 1]^{2} \\ a, & \text { otherwise. }\end{cases}
$$

The structures of $V_{a}^{(T, S)}$ is shown in Figure 1 .

The other group is $V_{T}^{S}$ and its dual, i.e., $V_{S}^{T}: L^{2} \rightarrow L$, which are proposed by Ümit Ertuğrul [11] and defined as

$$
V_{T}^{S}(x, y)= \begin{cases}S(x, y), & \text { if }(x, y) \in[0, a]^{2} \\ T(x, y), & \text { if }(x, y) \in[a, 1]^{2} \\ S(x \wedge a, y \wedge a), & \text { if }(x, y) \in[0, a] \times I_{a} \cup I_{a} \times[0, a] \cup I_{a} \times I_{a} \\ a, & \text { otherwise }\end{cases}
$$

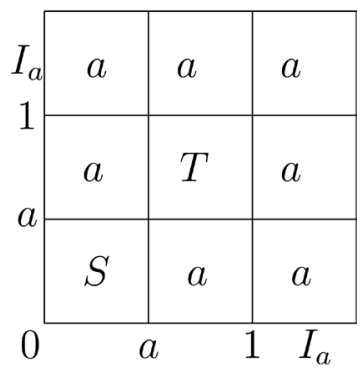

Figure 1. The frame of $V_{a}^{(T, S)}$. 
and

$$
V_{S}^{T}(x, y)= \begin{cases}S(x, y), & \text { if }(x, y) \in[0, a]^{2} \\ T(x, y), & \text { if }(x, y) \in[a, 1]^{2} \\ T(x \vee a, y \vee a), & \text { if }(x, y) \in[a, 1] \times I_{a} \cup I_{a} \times[a, 1] \cup I_{a} \times I_{a} \\ a, & \text { otherwise. }\end{cases}
$$

The structures of $V_{T}^{S}$ and $V_{S}^{T}$ are shown in Figure 2 and Figure 3, respectively. In these figures, we denote $S_{\wedge}=S(x \wedge a, y \wedge a)$ and $T_{\vee}=T(x \vee a, y \vee a)$.

\section{New Methods for Constructing Nullnorms on Bounded Lattices}

In order to reduce the complexity in the proof of associativity, we introduce the following proposition.

Proposition 3.1. ([17]) Let $S$ be a nonempty set and $A, B, C$ be subsets of $S$. Let $H$ be a commutative binary operation on $S$. Then $H$ is associative on $A \cup B \cup C$ if both of the following statements hold:

1) $H(H(x, y), z)=H(x, H(y, z))$ for all

$$
\begin{aligned}
(x, y, z) \in & (A, A, A) \cup(B, B, B) \cup(C, C, C) \cup(A, A, B) \cup(A, B, B) ; \\
\cup & (A, A, C) \cup(A, C, C) \cup(B, B, C) \cup(B, C, C)
\end{aligned}
$$

2) $H(H(x, y), z)=H(x, H(y, z))=H(H(x, z), y)$ for all $(x, y, z) \in(A, B, C)$.

Now, we introduce two construction methods which can be regard as generalizations of existing methods.

Theorem 3.2. Let $(L, \leq, 0,1)$ be a bounded lattice and $a \in L \backslash\{0,1\}$. Let

\begin{tabular}{|c|c|c|c|}
\hline$I_{a}$ & $S_{\wedge}$ & $a$ & $S_{\wedge}$ \\
\cline { 2 - 4 } & $a$ & $T$ & $a$ \\
\hline & $S$ & $a$ & $S_{\wedge}$ \\
\hline
\end{tabular}

Figure 2. The frame of $V_{T}^{S}$.

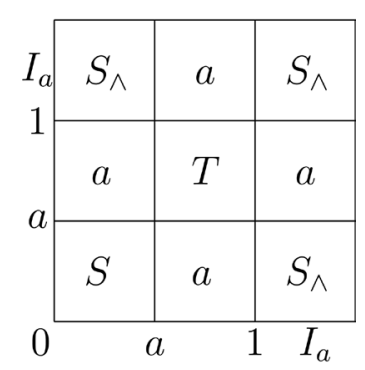

Figure 3. The frame of $V_{S}^{T}$. 
$T:[a, 1]^{2} \rightarrow[a, 1]$ be a t-norm on $[a, 1], S:[0, a]^{2} \rightarrow[0, a]$ be a t-conorm on $[0, a]$ and $R:[0, a]^{2} \rightarrow[0, a]$ be a t-subconorm on $[0, a]$. If $S \leq R$ and

$$
S(x, R(y, z))=R(R(x, y), z)=R(S(x, y), z) \text { forall } x, y, z \in[0, a],
$$

then $V_{T}^{S, R}: L^{2} \rightarrow L$ is a nullnorm on $L$ with the zero element $a$, where

$$
V_{T}^{S, R}(x, y)= \begin{cases}S(x, y), & \text { if }(x, y) \in[0, a]^{2} \\ T(x, y), & \text { if }(x, y) \in[a, 1]^{2} \\ R(x \wedge a, y \wedge a), & \text { if }(x, y) \in[0, a] \times I_{a} \cup I_{a} \times[0, a] \cup I_{a} \times I_{a} \\ a, & \text { otherwise. }\end{cases}
$$

Proof. The commutativity of $V_{T}^{S, R}$ can be proven directly based on its description. Similarly, we can express $V_{T}^{S, R}(x, 0)=S(x, 0)=x$ for all $x \in[0, a]$ and $V_{T}^{S, R}(x, 1)=T(x, 1)=x$ for all $x \in[a, 1]$.

Monotonicity: Let us prove that if $x \leq y$, then $V_{T}^{S, R}(x, z) \leq V_{T}^{S, R}(y, z)$ for all $z \in L$. If $x, y \in[0, a]$, or $x, y \in I_{a}$, or $x, y \in(a, 1]$, then it is clear that $U(x, z) \leq U(y, z)$ because $(x, z)$ and $(y, z)$ are in the same piece of $U$ and $U$ is monotonic in each piece. Moreover, $(x, y) \in(a, 1] \times[0, a] \cup I_{a} \times[0, a] \cup I_{a} \times(a, 1]$ contradicts the assumption that $x \leq y$. Therefore, there are only three cases left to consider, namely, $(x, y) \in[0, a] \times(a, 1], \quad(x, y) \in[0, a] \times I_{a}$, and

$(x, y) \in I_{a} \times(a, 1]$.

(I) Assume that $x \in[0, a]$ and $y \in(a, 1]$.

(i) If $z \in[0, a]$, then $V_{T}^{S, R}(x, z)=S(x, z)$ and $V_{T}^{S, R}(y, z)=a$. As

$S(x, z) \leq a$, we have $V_{T}^{S, R}(x, z) \leq V_{T}^{S, R}(y, z)$.

(ii) If $z \in(a, 1]$, then $V_{T}^{S, R}(x, z)=a$ and $V_{T}^{S, R}(y, z)=T(y, z)$. As

$a \leq T(y, z)$, we have $V_{T}^{S, R}(x, z) \leq V_{T}^{S, R}(y, z)$.

(iii) If $z \in I_{a}$, then $V_{T}^{S, R}(x, z)=R(x \wedge a, z \wedge a)$ and $V_{T}^{S, R}(y, z)=a$. As $R(x \wedge a, z \wedge a) \leq a$, we have $V_{T}^{S, R}(x, z) \leq V_{T}^{S, R}(y, z)$.

Therefore, $V_{T}^{S, R}(x, z) \leq V_{T}^{S, R}(y, z)$ holds for $(x, y) \in[0, a] \times[a, 1]$.

(II) Assume that $x \in[0, a]$ and $y \in I_{a}$ such that $x \leq y$.

(i) If $z \in[0, a]$, then $V_{T}^{S, R}(x, z)=S(x, z)$ and $V_{T}^{S, R}(y, z)=R(y \wedge a, z \wedge a)$.

As $S(x, z)=S(x \wedge a, z \wedge a) \leq R(x \wedge a, z \wedge a) \leq R(y \wedge a, z \wedge a)$, we have $V_{T}^{S, R}(x, z) \leq V_{T}^{S, R}(y, z)$.

(ii) If $z \in[a, 1]$, then $V_{T}^{S, R}(x, z)=a$ and $V_{T}^{S, R}(y, z)=a$, and thus $V_{T}^{S, R}(x, z) \leq V_{T}^{S, R}(y, z)$.

(iii) If $z \in I_{a}$, then $V_{T}^{S, R}(x, z)=R(x \wedge a, z \wedge a)$ and $V_{T}^{S, R}(y, z)=R(y \wedge a, z \wedge a)$. As $R(x \wedge a, z \wedge a) \leq R(y \wedge a, z \wedge a)$, we have $V_{T}^{S, R}(x, z) \leq V_{T}^{S, R}(y, z)$.

Therefore, $V_{T}^{S, R}(x, z) \leq V_{T}^{S, R}(y, z)$ holds for $(x, y) \in[0, a] \times I_{a}$.

(III) Assume that $x \in I_{a}$ and $y \in(a, 1]$ such that $x \leq y$.

(i) If $z \in[0, a]$, then $V_{T}^{S, R}(x, z)=R(x \wedge a, z \wedge a)$ and $V_{T}^{S, R}(y, z)=a$. As $R(x \wedge a, z \wedge a) \leq a$, we have $V_{T}^{S, R}(x, z) \leq V_{T}^{S, R}(y, z)$.

(ii) If $z \in[a, 1]$, then $V_{T}^{S, R}(x, z)=a$ and $V_{T}^{S, R}(y, z)=T(y, z)$. As $a \leq T(y, z)$, we have $V_{T}^{S, R}(x, z) \leq V_{T}^{S, R}(y, z)$.

(iii) If $z \in I_{a}$, then $V_{T}^{S, R}(x, z)=R(x \wedge a, z \wedge a)$ and $V_{T}^{S, R}(y, z)=a$. As 
$R(x \wedge a, z \wedge a) \leq a$, we have $V_{T}^{S, R}(x, z) \leq V_{T}^{S, R}(y, z)$.

Therefore, $V_{T}^{S, R}(x, z) \leq V_{T}^{S, R}(y, z)$ holds for $(x, y) \in I_{a} \times(a, 1]$.

Combining the above cases, we obtain that $V_{T}^{S, R}(x, z) \leq V_{T}^{S, R}(y, z)$ holds for $x, y, z \in L$ such that $x \leq y$. Therefore, $V_{T}^{S, R}$ is monotonic.

Associativity: It can be shown that $V_{T}^{S, R}\left(x, V_{T}^{S, R}(y, z)\right)=V_{T}^{S, R}\left(V_{T}^{S, R}(x, y), z\right)$ for all $x, y, z \in L$. By Proposition 3.1, We only need to consider the following cases:

(i) If $x, y, z \in[0, a]$, then since $S$ is associative, we have $V_{T}^{S, R}\left(V_{T}^{S, R}(x, y), z\right)=V_{T}^{S, R}\left(x, V_{T}^{S, R}(y, z)\right)$.

(ii) If $x, y, z \in[a, 1]$, then since $T$ is associative, we have $V_{T}^{S, R}\left(V_{T}^{S, R}(x, y), z\right)=V_{T}^{S, R}\left(x, V_{T}^{S, R}(y, z)\right)$.

(iii) If $x, y, z \in I_{a}$, then

$V_{T}^{S, R}\left(V_{T}^{S, R}(x, y), z\right)=V_{T}^{S, R}(R(x \wedge a, y \wedge a), z)=R(R(x \wedge a, y \wedge a), z \wedge a)$, $V_{T}^{S, R}\left(x, V_{T}^{S, R}(y, z)\right)=V_{T}^{S, R}(x, R(y \wedge a, z \wedge a))=R(x \wedge a, R(y \wedge a, z \wedge a))$. As $R$ is an associative function on $[0, a]$, we have $V_{T}^{S, R}\left(V_{T}^{S, R}(x, y), z\right)=V_{T}^{S, R}\left(x, V_{T}^{S, R}(y, z)\right)$.

(iv) If $x, y \in[0, a]$ and $z \in[a, 1]$, then $V_{T}^{S, R}\left(V_{T}^{S, R}(x, y), z\right)=V_{T}^{S, R}(S(x, y), z)=a$ and $V_{T}^{S, R}\left(x, V_{T}^{S, R}(y, z)\right)=V_{T}^{S, R}(x, a)=a$, and thus $V_{T}^{S, R}\left(V_{T}^{S, R}(x, y), z\right)=V_{T}^{S, R}\left(x, V_{T}^{S, R}(y, z)\right)$.

(v) If $x \in[0, a]$ and $y, z \in[a, 1]$, then $V_{T}^{S, R}\left(V_{T}^{S, R}(x, y), z\right)=a$ and $V_{T}^{S, R}\left(x, V_{T}^{S, R}(y, z)\right)=V_{T}^{S, R}(x, T(y, z))=a$. Thus $V_{T}^{S, R}\left(V_{T}^{S, R}(x, y), z\right)=V_{T}^{S, R}\left(x, V_{T}^{S, R}(y, z)\right)$.

(vi) If $x, y \in[0, a]$ and $z \in I_{a}$, then $V_{T}^{S, R}\left(V_{T}^{S, R}(x, y), z\right)=V_{T}^{S, R}(S(x, y), z)=R(S(x, y), z \wedge a)$ and $V_{T}^{S, R}\left(x, V_{T}^{S, R}(y, z)\right)=V_{T}^{S, R}(x, R(y \wedge a, z \wedge a))=S(x, R(y \wedge a, z \wedge a))$. It follows from (6) that $V_{T}^{S, R}\left(V_{T}^{S, R}(x, y), z\right)=V_{T}^{S, R}\left(x, V_{T}^{S, R}(y, z)\right)$.

(vii) If $x \in[0, a]$ and $y, z \in I_{a}$, then $V_{T}^{S, R}\left(V_{T}^{S, R}(x, y), z\right)=V_{T}^{S, R}(R(x \wedge a, y \wedge a), z)=R(R(x \wedge a, y \wedge a), z \wedge a) \quad$ and $V_{T}^{S, R}\left(x, V_{T}^{S, R}(y, z)\right)=V_{T}^{S, R}(x, R(y \wedge a, z \wedge a))=S(x, R(y \wedge a, z \wedge a))$. It follows from (6) that $V_{T}^{S, R}\left(V_{T}^{S, R}(x, y), z\right)=V_{T}^{S, R}\left(x, V_{T}^{S, R}(y, z)\right)$.

(viii) If $x, y \in[a, 1]$ and $z \in I_{a}$, then $V_{T}^{S, R}\left(V_{T}^{S, R}(x, y), z\right)=V_{T}^{S, R}(T(x, y), z)=a$ and $V_{T}^{S, R}\left(x, V_{T}^{S, R}(y, z)\right)=a$. Thus $V_{T}^{S, R}\left(V_{T}^{S, R}(x, y), z\right)=V_{T}^{S, R}\left(x, V_{T}^{S, R}(y, z)\right)$.

(ix) If $x \in[a, 1]$ and $y, z \in I_{a}$, then $V_{T}^{S, R}\left(V_{T}^{S, R}(x, y), z\right)=a$ and $V_{T}^{S, R}\left(x, V_{T}^{S, R}(y, z)\right)=V_{T}^{S, R}(x, R(y \wedge a, z \wedge a))=a$. Thus $V_{T}^{S, R}\left(V_{T}^{S, R}(x, y), z\right)=V_{T}^{S, R}\left(x, V_{T}^{S, R}(y, z)\right)$. 
(x) If $x \in[0, a], y \in(a, 1], \quad z \in I_{a}$, then $V_{T}^{S, R}\left(V_{T}^{S, R}(x, y), z\right)=a$, $V_{T}^{S, R}\left(x, V_{T}^{S, R}(y, z)\right)=a$ and $V_{T}^{S, R}\left(V_{T}^{S, R}(x, z), y\right)=V_{T}^{S, R}(R(x \wedge a, z \wedge a), y)=a$. Thus $V_{T}^{S, R}\left(V_{T}^{S, R}(x, y), z\right)=V_{T}^{S, R}\left(x, V_{T}^{S, R}(y, z)\right)=V_{T}^{S, R}\left(V_{T}^{S, R}(x, z), y\right)$.

From (i) to (x), we obtain that $V_{T}^{S, R}\left(x, V_{T}^{S, R}(y, z)\right)=V_{T}^{S, R}\left(V_{T}^{S, R}(x, y), z\right)$ for all $x, y, z \in L$ by Proposition 3.1. Therefore, $V_{T}^{S, R}$ is a nullnorm on $L$ with the zero element $a . \square$

Theorem 3.3. Let $(L, \leq, 0,1)$ be a bounded lattice and $a \in L \backslash\{0,1\}$. Let $T:[a, 1]^{2} \rightarrow[a, 1]$ be a t-norm on $[a, 1], F:[a, 1]^{2} \rightarrow[a, 1]$ be a t-subnorm on $[a, 1]$ and $S:[0, a]^{2} \rightarrow[0, a]$ be at-conorm on $[0, a]$. If $F \leq T$ and $T(x, F(y, z))=F(F(x, y), z)=F(T(x, y), z)$ for all $x, y, z \in L$, then $V_{S}^{T, F}: L^{2} \rightarrow L$ is a nullnorm on $L$ with the zero element $a$, where

$$
V_{S}^{T, F}(x, y)= \begin{cases}S(x, y), & \text { if }(x, y) \in[0, a]^{2} \\ T(x, y), & \text { if }(x, y) \in[a, 1]^{2} \\ F(x \vee a, y \vee a), & \text { if }(x, y) \in[a, 1] \times I_{a} \cup I_{a} \times[a, 1] \cup I_{a} \times I_{a} \\ a, & \text { otherwise. }\end{cases}
$$

Proof. This can be proved similarly as Theorem 3.2.

The structures of $V_{T}^{S, R}$ and $V_{S}^{T, F}$ from Formula (7) and Formula (8) are shown in Figure 4 and Figure 5, respectively. We denote $R_{\wedge}=R(x \wedge a, y \wedge a)$ and $F_{\vee}=F(x \vee a, y \vee a)$ in these figures.

Let $(L, \leq, 0,1)$ be a bounded lattice and $a \in L \backslash\{0,1\}$. Let $T:[a, 1]^{2} \rightarrow[a, 1]$ be a t-norm on $[a, 1], S:[0, a]^{2} \rightarrow[0, a]$ be a t-conorm on $[0, a]$. Taking $R(x, y)=S(x, y)$ in Formula (7), we obtain that

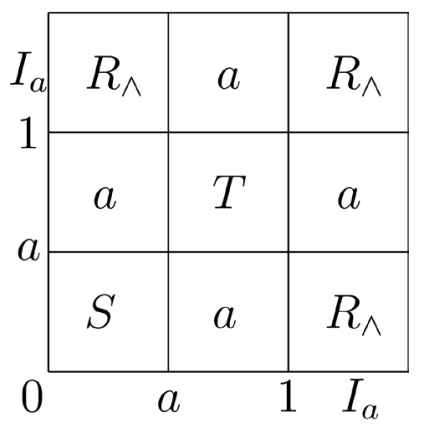

Figure 4. The frame of $V_{S}^{T, F}$.

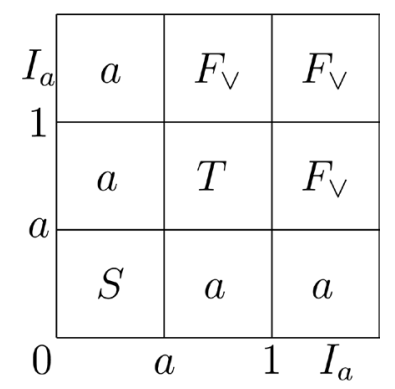

Figure 5. The frame of $V_{S}^{T, F}$. 


$$
V_{T}^{S, S}(x, y)= \begin{cases}S(x, y), & \text { if }(x, y) \in[0, a]^{2} \\ T(x, y), & \text { if }(x, y) \in[a, 1]^{2} \\ S(x \wedge a, y \wedge a), & \text { if }(x, y) \in[0, a] \times I_{a} \cup I_{a} \times[0, a] \cup I_{a} \times I_{a} \\ a, & \text { otherwise, }\end{cases}
$$

which is equal to $V_{T}^{S}(x, y)$ given by Formula (4).

Dually, taking $F(x, y)=T(x, y)$ in Formula (8), we obtain that

$$
V_{S}^{T, T}(x, y)= \begin{cases}S(x, y), & \text { if }(x, y) \in[0, a]^{2} \\ T(x, y), & \text { if }(x, y) \in[a, 1]^{2} \\ T(x \vee a, y \vee a), & \text { if }(x, y) \in[a, 1] \times I_{a} \cup I_{a} \times[a, 1] \cup I_{a} \times I_{a} \\ a, & \text { otherwise }\end{cases}
$$

which is equal to $V_{S}^{T}(x, y)$ given by Formula (5).

Taking $R(x, y)=a$ for all $(x, y) \in[0, a]^{2}$ in Formula (7), then

$$
V_{T}^{S, a}(x, y)= \begin{cases}S(x, y), & \text { if }(x, y) \in[0, a]^{2} \\ T(x, y), & \text { if }(x, y) \in[a, 1]^{2} \\ a, & \text { otherwise, }\end{cases}
$$

which is equal to $V_{a}^{(T, S)}$ given by Formula (3).

Taking $R(x, y)=a$ for all $(x, y) \in[0, a]^{2}$ in Formula (8), then it is clear that $V_{S}^{T, a}(x, y)$ also coincides with $V_{a}^{(T, S)}$, which is given by Formula (3). Therefore, the two methods proposed in this study are more generalized than the methods proposed previously by [10] [11]. Now we give an example to show that we can obtain new nullnorms by the construction methods proposed in this paper.

Example 3.4. Let $(L, \leq, 0,1)$ be a bounded lattice and let $a \in L \backslash\{0,1\}$.

(i) Let $T:[a, 1]^{2} \rightarrow[a, 1]$ be a t-norm on $[a, 1]$ and $b, c \in L$ be such that $c \leq b \leq a$. Let $S:[0, a]^{2} \rightarrow[0, a]$ and $R:[0, a]^{2} \rightarrow[0, a]$ be two functions on $[0, a]$ defined by

$$
S(x, y)= \begin{cases}x, & \text { if } y=0 \text { and } x \in[0, a] \\ y, & \text { if } x=0 \text { and } y \in[0, a] \\ x \vee y \vee c, & \text { if }(x, y) \in[0, a)^{2}\end{cases}
$$

and

$$
R(x, y)=x \vee y \vee b .
$$

Then $S$ is a t-conorm and $R$ is a t-subconorm on $[0, a]$. It is easy to verify $S \leq R$ and the condition (6) holds. Therefore,

$$
V_{1}(x, y)= \begin{cases}x, & \text { if } y=0 \text { and } x \in[0, a] \\ y, & \text { if } x=0 \text { and } y \in[0, a] \\ x \vee y \vee c, & \text { if }(x, y) \in(0, a]^{2} \\ T(x, y), & \text { if }(x, y) \in[a, 1]^{2} \\ (x \wedge a) \vee(y \wedge a) \vee b, & \text { if }(x, y) \in[0, a] \times I_{a} \cup I_{a} \times[0, a] \cup I_{a} \times I_{a} \\ a, & \text { otherwise }\end{cases}
$$


is a nullnorm on $L$ with the zero element a by Theorem 3.2.

(ii) Dually, let $S:[0, a]^{2} \rightarrow[0, a]$ be a t-conorm on $[0, a]$ and $j, k \in L$ be such that $a \leq j \leq k$. Then $V_{2}: L^{2} \rightarrow L$ is a nullnorm on $L$ with the zero element $a$ by Theorem 3.3, where

$$
V_{2}(x, y)= \begin{cases}x, & \text { if } y=1 \text { and } x \in[a, 1] \\ y, & \text { if } x=1 \text { and } y \in[a, 1] \\ x \wedge y \wedge k, & \text { if }(x, y) \in[a, 1)^{2} \\ S(x, y), & \text { if }(x, y) \in[0, a]^{2} \\ (x \vee a) \wedge(y \vee a) \wedge j, & \text { if }(x, y) \in[a, 1] \times I_{a} \cup I_{a} \times[a, 1] \cup I_{a} \times I_{a} \\ a, & \text { otherwise. }\end{cases}
$$

\section{Conclusion}

In this study, based on the existing constructions of nullnorms on $L$, we continue to study construction methods of nullnorms on bounded lattices. Two methods for obtaining nullnorms on $L$ are presented in this paper. Some examples were provided to show that the construction methods proposed in this paper generalized the methods presented in previous studies.

\section{Conflicts of Interest}

The authors declare no conflicts of interest regarding the publication of this paper.

\section{References}

[1] Schweizer, B. and Sklar, A. (1960) Statistical Metric Spaces. Pacific Journal of Mathematics, 10, 313-334. https://doi.org/10.2140/pjm.1960.10.313

[2] Mas, M., Mayor, G. and Torrens, J. (2002) The Modularity Condition for Uninorms and t-Operators. Fuzzy Sets and Systems, 126, 207-218. https://doi.org/10.1016/S0165-0114(01)00055-0

[3] Drewniak, J., Drygas, P. and Rak, E. (2008) Distributivity between Uninorms and Nullnorms. Fuzzy Sets and Systems, 159, 1646-1657. https://doi.org/10.1016/j.fss.2007.09.015

[4] Drygas, P. (2004) A Characterization of Idempotent Nullnorms. Fuzzy Sets and Systems, 145, 455-461. https://doi.org/10.1016/S0165-0114(03)00259-8

[5] Li, G., Liu, H. and Su, Y. (2015) On the Conditional Distributivity of Nullnorms over Uninorms. Information Sciences, 317, 157-169. https://doi.org/10.1016/j.ins.2015.04.040

[6] Xie, A. and Liu, H. (2013) On the Distributivity of Uninorms over Nullnorms. Fuzzy Sets and Systems, 211, 62-72. https://doi.org/10.1016/i.fss.2012.05.008

[7] Drygaś, P. (2015) Distributivity between Semi t-Operators and Semi Nullnorms. Fuzzy Sets and Systems, 126, 100-109. https://doi.org/10.1016/j.fss.2014.09.003

[8] Mas, M., Mesiar, R., Monserrat, M. and Torrens, J. (2005) Aggregation Operators with Annihilator. International Journal of General Systems, 34, 17-38. https://doi.org/10.1080/03081070512331318347

[9] Qin, F. and Zhao, B. (2005) The Distributive Equations for Idempotent Uninorms 
and Nullnorms. Fuzzy Sets and Systems, 155, 446-458.

https://doi.org/10.1016/j.fss.2005.04.010

[10] Karaçal, F., İnce, M.A. and Mesiar, R. (2015) Nullnorms on Bounded Lattices. Information Sciences, 325, 227-236. https://doi.org/10.1016/j.ins.2015.06.052

[11] Ertuğrul, Ü. (2018) Construction of Nullnorms on Bounded Lattices and an Equivalence Relation on Nullnorms. Fuzzy Sets and Systems, 334, 94-109.

https://doi.org/10.1016/j.fss.2017.07.020

[12] Lnce, M.A., Karaçal, F. and Mesiar, R. (2016) Medians and Nullnorms on Bounded Lattices. Fuzzy Sets and Systems, 289, 74-81.

https://doi.org/10.1016/j.fss.2015.05.015

[13] De Baets, B. and Mesiar, R. (1999) Triangular Norms on Product Lattices. Fuzzy Sets and Systems, 104, 61-75. https://doi.org/10.1016/S0165-0114(98)00259-0

[14] Klement, E.P., Mesiar, R. and Pap, E. (2000) Triangular Norms. Kluwer Academic Publishers, Dordrecht. https://doi.org/10.1007/978-94-015-9540-7

[15] Palmeira, E.S. and Bedregal, B.C. (2012) Extension of Fuzzy Logic Operators Defined on Bounded Lattices via Retractions. Computers \& Mathematics with Applications, 63, 1026-1038. https://doi.org/10.1016/j.camwa.2011.12.007

[16] Drygaś, P. (2004) Isotonic Operations with Zero Element in Bounded Lattices. In: Atanassov, K., Hryniewicz, O. and Kacprzyk, J., Eds., Soft Computing Foundations and Theoretical Aspect, EXIT, Warszawa, 181-190.

[17] Ji, W. (2021) Constructions of Uninorms on Bounded Lattices by Means of t-Subnorms and t-Subconorms. Fuzzy Sets and Systems, 403, 38-55.

https://doi.org/10.1016/j.fss.2019.12.003 